\title{
Associations between Features of External Ventricular Drain Management, Disposition, and Shunt Dependence
}

\author{
Corey Engel ${ }^{1} \quad$ Amanda L. Faulkner ${ }^{2, \odot} \quad$ David W. Van Wyck ${ }^{3} \quad$ Ali R. Zomorodi ${ }^{4} \quad$ Nicolas K. Kam King ${ }^{5}$ \\ Rachel A. Williamson Taylor ${ }^{6} \quad$ Claire E. Hailey ${ }^{7}$ Odera A. Umeano ${ }^{8} \quad$ David L. McDonagh ${ }^{9} \quad$ Yi-Ju Li ${ }^{10}$ \\ Michael L. James ${ }^{2,3, \odot}$ for the Duke Critical Care Outcomes Research Endeavors (C-CORE) group ${ }^{11}$
}

${ }^{1}$ Florida State University College of Medicine, Tallahassee, Florida, United States

2Department of Anesthesiology, Duke University, Durham, North Carolina, United States

${ }^{3}$ Department of Neurology, Duke University, Durham, North Carolina, United States

${ }^{4}$ Department of Neurosurgery, Duke University, Durham, North Carolina, United States

${ }^{5}$ Department of Neurosurgery, National Neuroscience Institute, Singapore, Singapore

${ }^{6}$ Department of Obstetrics and Gynecology, Beaumont Health, Royal Oak, Michigan, United States

${ }^{7}$ Department of Pediatrics, University of Chicago, Chicago, Illinois, United States
Address for correspondence Amanda L. Faulkner, MD, Department of Anesthesiology, Duke University Hospital, DUMC 3094, Durham, NC 27710, United States (e-mail: amanda.faulkner@uky.edu).

${ }^{8}$ Department of Internal Medicine, New Hanover Regional Medical Center, Wilmington, North Carolina, United States

9 UT Southwestern Departments of Anesthesiology and Pain Management, Neurology and Neurotherapeutics, and Neurological Surgery, Dallas, Texas, United States

${ }^{10}$ Department of Biostatistics \& Bioinformatics, Duke University, Durham, North Carolina, United States

${ }^{11}$ Department of Anesthesiology, Division of Critical Care, Duke University, Durham, North Carolina, United States

\author{
Abstract \\ Keywords \\ - ventriculostomy \\ - ventriculoperitoneal \\ shunt \\ - adult \\ - weaning \\ - drainage \\ - intracranial pressure
}

Background In the United States, nearly 25,000 patients annually undergo percutaneous ventriculostomy for the management of increased intracranial pressure with little consensus on extraventricular drain management. To characterize relationships between external ventricular drain management, permanent ventriculoperitoneal shunt placement, and hospital disposition, we hypothesized that patients requiring extended drainage would have greater association with ventriculoperitoneal shunt placement and unfavorable disposition.

Methods Adult patients admitted to the Duke University Hospital Neuroscience Intensive Care Unit between 2008 and 2010 with extraventricular drains were analyzed. A total of 115 patient encounters were assessed to determine relative impact of age, sex, days of extraventricular placement, weaning attempts, cerebrospinal fluid drainage volumes, Glasgow Coma Scale, and physician's experience on disposition at discharge and ventriculoperitoneal shunt placement. Univariate logistic regression was first used to test the effect of each variable on the outcome, followed by backward selection to determine a final multivariable logistic regression. Variables in the final model meeting $p<0.05$ were declared as significant factors for the outcome.

Results Increased extraventricular drain duration (odds ratio $[\mathrm{OR}]=1.17$, confidence interval $[\mathrm{Cl}]=1.05-1.30, p=0.0049$ ) was associated with ventriculoperitoneal shunt placement, while older age $(\mathrm{OR}=1.05, \mathrm{Cl}=1.02-1.08, p=0.0027)$ and less physician extraventricular drain management experience $(O R=4.04, C l=1.67-9.79, p=0.0020)$ were associated with unfavorable disposition.

Conclusion In a small cohort, exploratory analyses demonstrate potentially modifiable factors are associated with important clinical outcomes. These findings warrant further study to refine how such factors affect patient outcomes.
Published online June 1, 2020
DOI https://doi.org/

$10.1055 / \mathrm{s}-0040-1710410$

ISSN 2348-0548.
(C2020. Indian Society of Neuroanaesthesiology and Critical Care.

This is an open access article published by Thieme under the terms of the Creative Commons Attribution-NonDerivative-NonCommercial-License, permitting copying and reproduction so long as the original work is given appropriate credit. Contents may not be used for commercial purposes, or adapted, remixed, transformed or built upon. (https://creativecommons.org/licenses/by-nc-nd/4.0/).

Thieme Medical and Scientific Publishers Pvt. Ltd. A-12, 2nd Floor, Sector 2, Noida-201301 UP, India 


\section{Introduction}

In the United States alone, nearly 25,000 patients annually undergo percutaneous ventriculostomy for the management of increased intracranial pressure (ICP). ${ }^{1}$ The catheters currently employed for external ventricular drains (EVDs) have been used for decades with infrequent complications $^{2-5}$; yet, despite the frequency of the use of EVD in contemporary neurocritical care, little consensus on drain management exists.

Most EVD management philosophies aim to maintain functionality of the intrinsic cerebrospinal fluid (CSF) reabsorption mechanism at the level of the arachnoid granulations, thus eliminating the need for a permanent CSF-diverting shunt. Extant literature is scant and conflicting regarding the optimal EVD open-closed setting, pressure, and weaning strategy. For example, in the instance of subarachnoid hemorrhage (SAH), EVDs may be left continuously open, maintaining a low drain pressure to promote external flow of blood products that might otherwise impede resorption of CSF through arachnoid granulations. Alternatively, EVDs may be left closed and only opened to drain above a defined threshold ICP as an attempt to promote natural CSF flow and resorption.

Further, in patients with proven hydrocephalus requiring permanent CSF diversion via ventriculoperitoneal (VP) shunting, little consensus is available regarding associations between EVD management strategies and the likelihood of requiring permanent CSF diversion. ${ }^{6-8}$ The aim of this study was to characterize relationships between features of EVD management, permanent VP shunt placement, and hospital disposition. The hypothesis was that patients requiring extended low-pressure drainage or failed multiple EVD weaning attempts would have greater association with VP shunt placement and unfavorable disposition.

\section{Methods}

\section{Study Population}

After obtaining Duke University Institutional Review Board approval, retrospective review of the Duke University Hospital Neurosciences intensive care unit (NICU) database, which catalogs all NICU admissions, identified 136 consecutive adult patients with an EVD between September 2008 and May 2010. Patients were excluded if EVD placement occurred prior to admission to the Duke University Hospital (i.e., prior to transfer), a VP shunt was already in situ at the time of admission, or another intracranial monitoring device was utilized concurrently, since data from the device may have altered a patient's EVD management. A total of 21 patients were excluded, leaving 115 patient records for analysis.

\section{Determination of EVD Management Features}

EVD drainage pressure (in centimeters of water $\left[\mathrm{cm} \mathrm{H}_{2} \mathrm{O}\right]$ ) was obtained from each patient's morning neurocritical care progress note. Amount of CSF collected each day was not uniformly measured and, therefore, not available for analysis. EVD drainage duration was defined as the number of progress notes documenting EVD in situ after placement; similarly, EVD "clamping" was defined as documentation of an explicit statement in the progress notes and no CSF drainage. The Duke University Hospital NICU standard of care for EVD clamping was to not allow CSF drainage unless there is an evidence of neurological deterioration. Days on which the EVD was clamped were counted toward total drainage duration, while the days that the EVD was removed did not. "Low pressure" drainage was defined as an EVD setting at $10 \mathrm{~cm}$ $\mathrm{H}_{2} \mathrm{O}$ or less; the percentage of days each patient spent at low pressure drainage was used in statistical modeling.

Since no explicit definition of "weaning attempt" existed in patient records, a standardized definition was developed to quantify the number of these attempts. For this study, on the third day after the EVD was placed, EVDs were considered to be undergoing weaning. If EVD drainage pressure was increased at least every third day until EVD removal, no additional weaning attempts were said to occur. Each time EVD pressure was decreased or held constant for three consecutive days after the third day from EVD placement, then the patient was considered to have failed a weaning attempt. Clamping did not contribute to the number of weaning attempts (i.e., the patient could have any number of consecutive days with a clamped EVD without being considered to fail a weaning attempt).

Since neurosurgeons are primarily responsible for EVD management at Duke University Hospital, "EVD experience" of neurosurgeons was dichotomized at the management of greater than or less than or equal to 10 EVDs in our sample. Two of the 22 neurosurgeons managed $>10$ EVDs in our sample, both of whom were fellowship-trained vascular neurosurgeons.

\section{Outcome Variables}

The primary outcome was the need for permanent CSF diversion surgery. The positive VP shunt group included any subject undergoing VP shunt placement during the first year of follow-up after EVD placement, while the negative VP shunt group did not require permanent CSF diversion during that time. Finally, favorable disposition was defined as discharge to home, home health care, or acute rehabilitation. Unfavorable disposition was defined as discharge to skilled nursing facility, long-term care, or death during the inpatient stay.

\section{Statistical Analyses}

VP shunt placement (positive vs. negative) and disposition at discharge (favorable vs. unfavorable) were the two binary outcomes assessed. Summary statistics were computed as frequencies (percentages) for categorical variables and mean (standard deviation) for continuous variables for each outcome. Logistic regression was used to determine the relative impact of our various input variables on both outcomes, respectively. First, a univariate model was performed for each of the following variables: age, sex, days of EVD drainage, number of weaning attempts, percentage of days at low drainage, presenting Glasgow Coma Scale (GCS), presence of SAH on admission, and EVD experience. We selected variables with $p<0.10$ to form an initial 
multivariable logistic regression model. We then performed backward selection to remove the least significant variable in each iteration until a model reaching the minimum Akaike information criterion (AIC) as the final multivariable logistic regression model for the outcome of interest. A $p$-value of 0.05 was used as our threshold for statistical significance, and odds ratio (OR) and its 95\% confidence interval were reported for each variable. SAS version 9.4 (SAS Inc., Cary North Carolina, United States) was used for all data management and model creation. Additionally, the concordance statistic as well as the Hosmer and Lemeshow Goodness-of-Fit were performed to assess predictive accuracy and model fit.

\section{Results}

The final cohort used for analyses is described in - Table 1. The mean age was 52.3 years and $41.7 \%$ of subjects were female. $61.7 \%$ of the cohort presented with SAH on admission and $33.9 \%$ of patients experienced inpatient mortality. On average, EVDs were used for CSF diversion for 12 days, with $21.9 \%$ of patients requiring permanent CSF diversion.
Results for univariate and multivariable regression models are presented in -Table 2 for unfavorable disposition and -Table 3 for VP shunt placement. Age, initial GCS, two EVD weaning attempts, EVD experience, and EVD duration met the $p<0.1$ criteria from the univariate analysis for unfavorable disposition ( - Table 2 ). The final multivariable model showed that age $(\mathrm{OR}=1.05$, $95 \%$ confidence interval $[\mathrm{CI}]=1.02-1.08, p=0.0027)$ and EVD experience $(\mathrm{OR}=4.04,95 \% \mathrm{CI}=1.67-9.79, p=0.0020)$ were significantly associated with unfavorable disposition. With a concordance of 0.75 , the full model could predict the outcome for $75.5 \%$ and the Hosmer and Lemeshow test was not significant $(p>$ Chi-square $=0.3180)$. As for VP shunt placement outcome, five variables had $p<0.01$ in the univariate analysis; however, AIC reached minimum with initial GCS, EVD duration, and three more EVD weaning attempts (-Table 3 ). The final multivariable model showed that $\mathrm{EVD}$ duration $(\mathrm{OR}=1.17,95 \% \mathrm{CI}=1.05-1.30$, $p<0.0049$ ) increases the odds of requiring VP shunt placement. Similarly, the full model demonstrated $81.8 \%$ predictability, with the Hosmer and Lemeshow test suggesting adequate model fit $(p>$ Chi-square $=0.0797)$.

Table 1 Total study cohort dichotomized by disposition and VP shunt requirement

\begin{tabular}{|c|c|c|c|c|c|}
\hline & $\begin{array}{l}\text { Total } \\
N=115\end{array}$ & $\begin{array}{l}\text { Favorable } \\
\text { disposition } \\
N=66\end{array}$ & $\begin{array}{l}\text { Unfavorable } \\
\text { disposition } \\
N=49\end{array}$ & $\begin{array}{l}\text { No VP shunt } \\
N=90\end{array}$ & $\begin{array}{l}\text { VP shunt } \\
N=25\end{array}$ \\
\hline Age, y, mean (SD) & $52.3(15.4)$ & $48.8(13.9)$ & $57.0(16.1)$ & $51.9(15.9)$ & $54.0(13.3)$ \\
\hline Female, $n(\%)$ & $48(41.7 \%)$ & $31(47.0 \%)$ & $17(34.7 \%)$ & $38(42.2 \%)$ & $10(40.0 \%)$ \\
\hline \multirow{2}{*}{$\begin{array}{l}\text { Admission GCS, mean (SD) } \\
\text { median }\end{array}$} & $9.5(4.5)$ & $10.1(4.6)$ & $8.7(4.2)$ & $9.1(4.5)$ & $11.0(4.4)$ \\
\hline & 10.0 & 10.0 & 7.0 & 8.0 & 14.0 \\
\hline Presence of SAH, $n(\%)$ & $71(61.7 \%)$ & $43(65.2 \%)$ & $28(57.1 \%)$ & $53(58.9 \%)$ & $18(72.0 \%)$ \\
\hline EVD duration, $d$, mean (SD) & $12.0(6.9)$ & $12.9(7.0)$ & $10.7(6.6)$ & $10.3(4.5)$ & $17.9(10.3)$ \\
\hline \multicolumn{6}{|l|}{ EVD weaning attempts, $n$ (\%) } \\
\hline Missing & $2(1.7 \%)$ & $1(. \%)$ & $1(. \%)$ & $1(. \%)$ & $1(. \%)$ \\
\hline 1 & $56(48.7 \%)$ & $28(43.1 \%)$ & $28(58.3 \%)$ & $51(57.3 \%)$ & $5(20.8 \%)$ \\
\hline 2 & $36(31.3 \%)$ & $25(38.5 \%)$ & $11(22.9 \%)$ & $29(32.6 \%)$ & $7(29.2 \%)$ \\
\hline $3+$ & $21(18.5 \%)$ & $12(18.5 \%$ & $9(18.8 \%$ & $9(10.1 \%)$ & $12(50.0 \%)$ \\
\hline $\begin{array}{l}\text { Low pressure drainage, } d \text {, } \\
\text { mean (SD) }\end{array}$ & $0.5(0.3)$ & $0.5(0.3)$ & $0.4(0.4)$ & $0.4(0.3)$ & $0.6(0.3)$ \\
\hline $\begin{array}{l}\text { Less physician EVD } \\
\text { experience, }<11 \mathrm{EVDs}, n \text { (\%) }\end{array}$ & $58(50.4 \%)$ & $26(39.4 \%)$ & $32(65.3 \%)$ & $50(55.6 \%)$ & $8(32.0 \%)$ \\
\hline $\begin{array}{l}\text { Permanent CSF diversion, } \\
n(\%)\end{array}$ & $25(21.9 \%)$ & & & & \\
\hline Inpatient mortality, n (\%) & $39(33.9 \%)$ & & & & \\
\hline Poor disposition, $n(\%)$ & $49(42.6 \%)$ & & & & \\
\hline
\end{tabular}

Abbreviations: CSF, cerebrospinal fluid; EVD, extraventricular drain; GCS, Glasgow Coma Scale; SAH, subarachnoid hemorrhage; SD, standard deviation; VP, ventriculoperitoneal.

Note: Favorable disposition includes discharge to home, home health care, or acute rehabilitation. Unfavorable outcomes include discharge to a skilled nursing facility, long-term care, or death during the inpatient stay. VP shunt group includes any patient receiving VP shunt placement up to 1 year after initial EVD placement. 
Table 2 Logistic regression for associations with unfavorable disposition

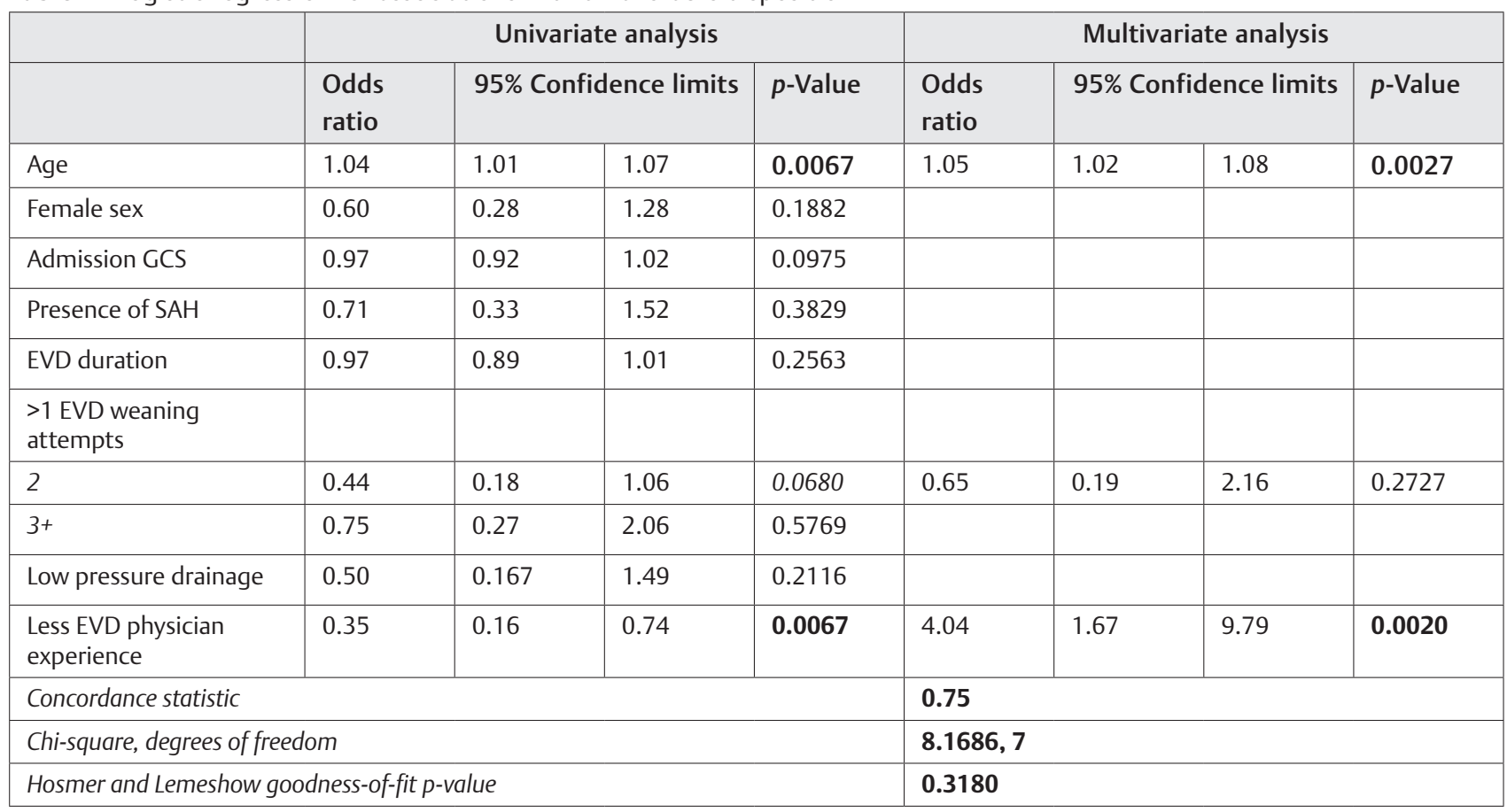

Abbreviations: EVD, extraventricular drain; GCS, Glasgow Coma Scale; SAH, subarachnoid hemorrhage.

Note: Logistic regressions for associations with unfavorable disposition. After univariate analysis, multivariate analysis was performed using backward selection with Akaike Information Criterion (AIC). The initial model included all variables with $p<0.10$; in each iteration the least significant variable was removed and the final model was selected when the AIC reached minimum. Bolded p-values are significant.

Table 3 Logistic regressions for associations with permanent CSF diversion

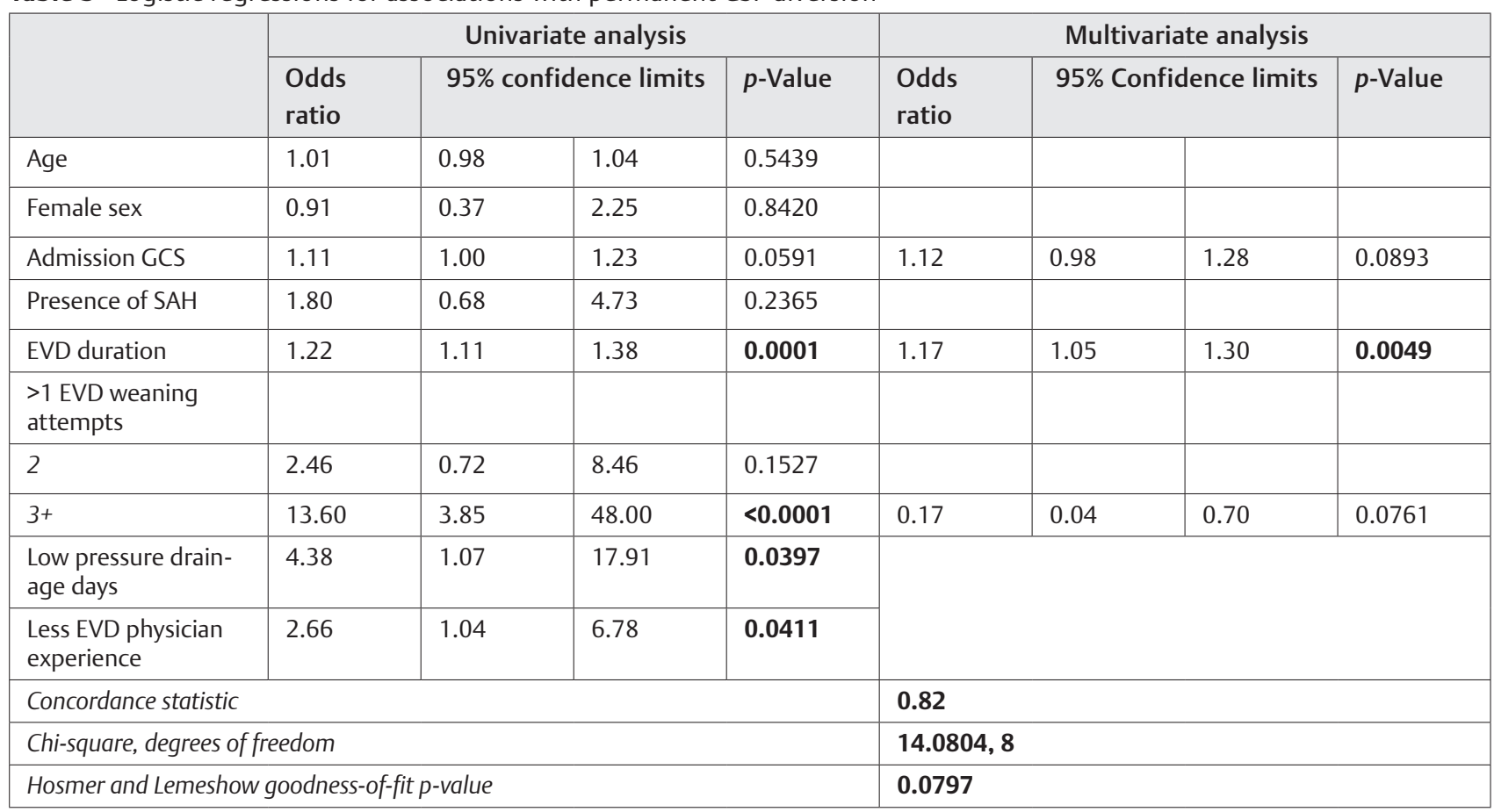

Abbreviations: EVD, extraventricular drain; GCS, Glasgow Coma Scale; SAH, subarachnoid hemorrhage; VP, ventriculoperitoneal.

Note: Logistic regressions for associations with VP shunt requirement. After univariate analysis, multivariate analysis was performed using backward selection with Akaike Information Criterion criteria. The initial model included all variables with $p<0.10$; in each iteration the least significant variable was removed and the final model was selected when the AIC reached minimum. Bolded $p$-values are significant. 


\section{Discussion}

EVD placement is often a life-saving intervention for patients with hydrocephalus and/or elevated ICP. While existing literature has addressed EVD-associated complications, fewer studies have examined predictors for discharge disposition. Little consensus exists for predicting VP shunt insertion following EVD placement despite previous studies assessing the impact of age, sex, ${ }^{9,10}$ EVD management, ${ }^{6-8}$ and other variables on VP shunt requirement. Novel grading scales ${ }^{11-14}$ have been developed with the aim of predicting shunt requirement; however, none have been independently validated. Within our cohort, older patients and those managed by physicians with less EVD experience were more likely to have unfavorable discharge disposition. Only the number of days with an EVD in place was associated with permanent CSF diversion.

\section{Patient Age}

In patients with EVDs, our results align with previously described findings that older patients with EVD-treated conditions are more likely to have a poor outcome, notably severe disability, or death. ${ }^{15-18}$ Notably, for the present study, age predicted discharge disposition but not the need for VP shunt. As an immutable patient factor, patient age should factor into clinical decision making regarding the risk/benefit analysis of EVD placement. Interestingly, patient age was not associated with permanent CSF diversion in our cohort. Though, other confounders, such as mortality and poor recovery, may drive the lack of association. The median age and range over which both poor disposition and VP shunt placement occurred were similar in our cohort, implying that it is unlikely that this finding is attributable to selection bias.

\section{Physician Experience with EVDs}

In the present cohort, discharge disposition was associated with EVDs managed by physicians with more EVD experience. Characterizing relevant physician experience is challenging, especially specific to disease management. While studies have attempted to characterize physician experience by age ${ }^{19}$ and years in practice, ${ }^{20}$ substantial limitations associated with these measures led us to develop a procedure-specific, quantifiable measure of experience. Physicians who managed eleven or more EVDs in our dataset (approximately 9.5\%) were considered more experienced in managing EVDs, while physicians who managed ten or fewer were deemed less experienced. While all the 23 physicians in the present study were board-certified neurosurgeons, two fell into the more experienced category and were both later identified as the two fellowship-trained vascular neurosurgeons. Conversely, physicians characterized as less experienced with EVD management included general neurosurgeons and spine surgeons. While these results cannot be used to recommend EVD management by one physician type over another, correlations between physician experience and EVD outcomes are significant and novel. Although, our study design does not enable us to decipher specific factors that enhance clinical competency (e.g., specialized vascular neurosurgery training, increased clinician comfort or confidence, etc.), our result suggests that having fewer physicians manage a greater number of EVD cases may positively affect patient outcomes.

\section{EVD Management Techniques}

As highlighted by Chung et al, ${ }^{21}$ variation in EVD management techniques exists both across and within institutions. Similarly, in the present cohort EVD management techniques varied (i.e., draining at high pressure vs. draining at low pressure). Nevertheless, neither technique was associated with disposition or permanent CSF diversion in multivariate analyses. In their single-center clamp trial study, Ascanio et $\mathrm{a}^{22}$ found that a significant percentage of patients passed their second wean attempt without requiring a shunt. Interestingly, while EVD management did not affect outcomes, increased EVD duration was associated with the need for permanent CSF diversion. While this association makes intuitive sense, EVD management did not correlate with EVD duration, so future work is required to further characterize.

\section{Limitations/Future Work}

Our study is limited by its retrospective design and use of a single institution cohort; data collection was limited to what was available. Patients were not randomized for EVD management technique or to physician experience, and progression to permanent CSF shunting was determined by the judgment of individual physicians without a defined criteria or protocol. Lack of standardization resulted in reasonable, yet arbitrary, definitions of variables with high likelihood to impact patient outcomes (e.g., weaning attempts). Finally, the analyses performed are intended to be hypothesis generating, may not have been appropriately powered for the multiple comparisons, and require validation in a separate cohort. The present findings and their limitations highlight the need to fully define variables that predict patient disposition and need for permanent CSF diversion. Thus, prospective, multi-institutional study needs to be performed. Data from such a study would identify modifiable factors for the development of therapeutic interventions. Further, addressing the scarcity of information should lead to the data generation necessary for evidence-based guidelines development to optimize EVD management, with careful attention paid to EVD weaning.

\section{Conclusion}

The present cohort found that potentially modifiable factors (i.e., physician's experience with EVD management and duration of EVD placement) were associated with important clinical outcomes (i.e., favorable discharge disposition and permanent CSF shunting). Age, an immutable patient factor, was also associated with discharge disposition. While interesting, these findings require replication and further refinement on how such factors affect patient outcome.

\section{Conflict of Interest}

None declared. 


\section{Acknowledgment}

The authors would like to acknowledge Kathy Gage for assistance with editing.

\section{References}

1 Sekula RF, Cohen DB, Patek PM, Jannetta PJ, Oh MY. Epidemiology of ventriculostomy in the United States from 1997 to 2001. Br J Neurosurg 2008;22(2):213-218

2 Robinson F. An apparatus for continuous ventricular drainage and intraventricular therapy. J Neurosurg 1948;5(3):320-323

3 Bering EA Jr. A simplified apparatus for constant ventricular drainage. J Neurosurg 1951;8(4):450-452

4 Friedman WA, Vries JK. Percutaneous tunnel ventriculostomy. Summary of 100 procedures. J Neurosurg 1980;53(5):662-665

5 Kakarla UK, Kim LJ, Chang SW, Theodore N, Spetzler RF. Safety and accuracy of bedside external ventricular drain placement. Neurosurgery 2008;63(suppl 1) :ONS162-ONS166, discussion ONS166-ONS167

6 Lewis A, Taylor Kimberly W. Prediction of ventriculoperitoneal shunt placement based on type of failure during external ventricular drain wean. Clin Neurol Neurosurg 2014;125:109-113

7 Lewis A, Irvine H, Ogilvy C, Kimberly WT. Predictors for delayed ventriculoperitoneal shunt placement after external ventricular drain removal in patients with subarachnoid hemorrhage. Br J Neurosurg 2015;29(2):219-224

8 Lewis A, Kimberly TW. A retrospective analysis of cerebrospinal fluid drainage volume in subarachnoid hemorrhage and the need for early or late ventriculoperitoneal shunt placement. J Neurosurg Sci 2016;60(3):289-295

9 Dorai Z, Hynan LS, Kopitnik TA, Samson D. Factors related to hydrocephalus after aneurysmal subarachnoid hemorrhage. Neurosurgery 2003;52(4):763-769, discussion 769-771

10 Little AS, Zabramski JM, Peterson M, et al. Ventriculoperitoneal shunting after aneurysmal subarachnoid hemorrhage: analysis of the indications, complications, and outcome with a focus on patients with borderline ventriculomegaly. Neurosurgery 2008;62(3):618-627, discussion 618-627

11 Jabbarli R, Bohrer AM, Pierscianek D, et al. The CHESS score: a simple tool for early prediction of shunt dependency after aneurysmal subarachnoid hemorrhage. Eur J Neurol 2016;23(5): 912-918
12 Diesing D, Wolf S, Sommerfeld J, Sarrafzadeh A, Vajkoczy P, Dengler NF. A novel score to predict shunt dependency after aneurysmal subarachnoid hemorrhage. J Neurosurg 2018;128(5):1273-1279

13 Chan M, Alaraj A, Calderon M, et al. Prediction of ventriculoperitoneal shunt dependency in patients with aneurysmal subarachnoid hemorrhage. J Neurosurg 2009;110(1):44-49

14 Miller C, Tsivgoulis G, Nakaji P. Predictors of ventriculoperitoneal shunting after spontaneous intraparenchymal hemorrhage. Neurocrit Care 2008;8(2):235-240

15 Degos V, Gourraud PA, Tursis VT, et al. Elderly age as a prognostic marker of 1-year poor outcome for subarachnoid hemorrhage patients through its interaction with admission hydrocephalus. Anesthesiology 2012;117(6):1289-1299

16 Lanzino G, Kassell NF, Germanson TP, et al. Age and outcome after aneurysmal subarachnoid hemorrhage: why do older patients fare worse? J Neurosurg 1996;85(3):410-418

17 Rosengart AJ, Schultheiss KE, Tolentino J, Macdonald RL. Prognostic factors for outcome in patients with aneurysmal subarachnoid hemorrhage. Stroke 2007;38(8):2315-2321

18 Rinaldo L, Rabinstein AA, Lanzino G. Elderly age associated with poor functional outcome after rupture of anterior communicating artery aneurysms. J Clin Neurosci 2016;34:108-111

19 Tsugawa Y, Newhouse JP, Zaslavsky AM, Blumenthal DM, Jena AB. Physician age and outcomes in elderly patients in hospital in the US: observational study. BMJ 2017;357:j1797

20 Goodwin JS, Salameh H, Zhou J, Singh S, Kuo YF, Nattinger AB. Association of hospitalist years of experience with mortality in the hospitalized medicare population. JAMA Intern Med 2018;178(2):196-203

21 Chung DY, Leslie-Mazwi TM, Patel AB, Rordorf GA. Management of external ventricular drains after subarachnoid hemorrhage: a multi-institutional survey. Neurocrit Care 2017;26(3): 356-361

22 Ascanio LC, Gupta R, Adeeb N, et al. Relationship between external ventricular drain clamp trials and ventriculoperitoneal shunt insertion following nontraumatic subarachnoid hemorrhage: a single-center study. J Neurosurg 2018;130(3): 956-962 